\title{
SOME RESULTS AND PROBLEMS FOR ANISOTROPIC RANDOM WALKS ON THE PLANE
}

Endre Csáki, Antónia Földes, and Pál Révész

\begin{abstract}
This is an expository paper on the asymptotic results concerning path behaviour of the anisotropic random walk on the two-dimensional square lattice $\mathbb{Z}^{2}$. In recent years Miklós and the authors of the present paper investigated the properties of this random walk concerning strong approximations, local times and range. We give a survey of these results together with some further problems.
\end{abstract}

Key words: anisotropic random walk; strong approximation; 2-dimensional Wiener process; local time; laws of the iterated logarithm.

Mathematics Subject Classification (2010): Primary 60F17, 60G50; Secondary 60F15, 60J10, 60J65

\section{Introduction}

We consider random walks on the square lattice $\mathbb{Z}^{2}$ with possibly unequal symmetric horizontal and vertical step probabilities, so that these probabilities can

Endre Csáki

Alfréd Rényi Institute of Mathematics, Hungarian Academy of Sciences,

Budapest, P.O.B. 127, H-1364, Hungary,

e-mail: csaki.endre@ renyi.mta.hu

Antónia Földes

Department of Mathematics, College of Staten Island,

CUNY, 2800 Victory Blvd., Staten Island, New York 10314, U.S.A.

e-mail: antonia.foldes@csi.cuny.edu

Pál Révész

Institut für Statistik und Wahrscheinlichkeitstheorie,

Technische Universität Wien, Wiedner Hauptstrasse 8-10/107

A-1040 Vienna, Austria,

e-mail: revesz.paul@ renyi.mta.hu 
only depend on the value of the vertical coordinate. In particular, if such a random walk is situated at a site on the horizontal line $y=j \in \mathbb{Z}$, then at the next step it moves with probability $p_{j}$ to either vertical neighbor, and with probability $1 / 2-p_{j}$ to either horizontal neighbor. More formally, consider the random walk $\left\{\mathbf{C}(N)=\left(C_{1}(N), C_{2}(N)\right) ; N=0,1,2, \ldots\right\}$ on $\mathbb{Z}^{2}$ with the transition probabilities

$$
\begin{array}{r}
\mathbf{P}(\mathbf{C}(N+1)=(k+1, j) \mid \mathbf{C}(N)=(k, j)) \\
=\mathbf{P}(\mathbf{C}(N+1)=(k-1, j) \mid \mathbf{C}(N)=(k, j))=\frac{1}{2}-p_{j}, \\
\mathbf{P}(\mathbf{C}(N+1)=(k, j+1) \mid \mathbf{C}(N)=(k, j)) \\
=\mathbf{P}(\mathbf{C}(N+1)=(k, j-1) \mid \mathbf{C}(N)=(k, j))=p_{j},
\end{array}
$$

for $(k, j) \in \mathbb{Z}^{2}, N=0,1,2, \ldots$ We assume throughout the paper that $0<p_{j} \leq 1 / 2$ and $\min _{j \in \mathbb{Z}} p_{j}<1 / 2$. Unless otherwise stated we assume also that $\mathbf{C}(0)=(0,0)$. This model has a number of physical applications and the topic has a broad literature. We refer to Silver et al. [28], Seshadri et al. [26], Shuler [27], Westcott [30], where certain properties of this random walk were studied under various conditions. Heyde [14] proved an almost sure approximation for $C_{2}(\cdot)$ under the condition

$$
n^{-1} \sum_{j=1}^{n} p_{j}^{-1}=2 \gamma+o\left(n^{-\eta}\right), \quad n^{-1} \sum_{j=1}^{n} p_{-j}^{-1}=2 \gamma+o\left(n^{-\eta}\right)
$$

as $n \rightarrow \infty$ for some constants $\gamma, 1<\gamma<\infty$ and $1 / 2<\eta<\infty$.

Heyde et al. [16] treated the case when conditions similar to (1.2) are assumed but $\gamma$ can be different for the two parts of (1.2) and obtained almost sure convergence to the so-called oscillating Brownian motion. In Heyde [15] limiting distributions were given for $\mathbf{C}(\cdot)$ under the condition (1.2) but without remainder. Den Hollander [12] proved strong approximations for $\mathbf{C}(\cdot)$ in the case when $p_{j}$-s are random variables with values $1 / 4$ and $1 / 2$. Roerdink and Shuler [25] proved some asymptotic properties, including local limit theorems, under certain conditions. For more detailed history see [12].

First we give a general construction and discuss the issue of recurrence and transience of this random walk. In Section 2 we discuss strong approximations of the random walk $\{\mathbf{C}(N), N=0,1, \ldots\}$. Section 3 treats the local time and in Section 4 some results on the range will be given.

\subsection{General construction}

Suppose that in a probability space we have two independent simple symmetric random walks, i.e.,

$$
S_{1}(n), n=0,1,2, \ldots, \quad S_{2}(n), n=0,1,2, \ldots,
$$


where $S_{1}(0)=S_{2}(0)=0, S_{i}(\cdot)$ are sums of i.i.d. random variables each taking the values 1 and -1 with probability $1 / 2$. The local times of $S_{i}$ are defined by

$$
\xi_{i}(j, n)=\#\left\{0 \leq k \leq n: S_{i}(k)=j\right\}, \quad j \in \mathbb{Z}, \quad n=0,1,2, \ldots
$$

Moreover, on the same probability space we have a double array of independent geometric random variables

$$
G_{i}^{(j)}, i=1,2, \ldots, j \in \mathbb{Z}
$$

with distribution

$$
\mathbf{P}\left(G_{i}^{(j)}=k\right)=2 p_{j}\left(1-2 p_{j}\right)^{k}, k=0,1,2, \ldots
$$

We now construct our walk $\mathbf{C}(N)$ as follows. We will take all the horizontal steps consecutively from $S_{1}(\cdot)$ and all the vertical steps consecutively from $S_{2}(\cdot)$. First we will take some horizontal steps from $S_{1}(\cdot)$, then exactly one vertical step from $S_{2}(\cdot)$, then again some horizontal steps from $S_{1}(\cdot)$ and exactly one vertical step from $S_{2}(\cdot)$, and so on. Now we explain how to get the number of horizontal steps on each occasion. Consider our walk starting from the origin proceeding first horizontally $G_{1}^{(0)}$ steps (note that $G_{1}^{(0)}=0$ is possible with probability $2 p_{0}$ ), after which it takes exactly one vertical step, arriving either to the level 1 or -1 , where it takes $G_{1}^{(1)}$ or $G_{1}^{(-1)}$ horizontal steps (which might be no steps at all) before proceeding with another vertical step. If this step carries the walk to the level $j$, then it will take $G_{1}^{(j)}$ horizontal steps, if this is the first visit to level $j$, otherwise it takes $G_{2}^{(j)}$ horizontal steps. In general, if we finished the $k$-th vertical step and arrived to the level $j$ for the $i$-th time, then it will take $G_{i}^{(j)}$ horizontal steps.

In this paper $N$ will denote the number of steps of the walk out of which $H_{N}$ denotes the number of horizontal steps and $V_{N}=n$ the number of vertical steps, i.e., $H_{N}+V_{N}=N$. Then clearly

$$
\mathbf{C}(N)=\left(C_{1}(N), C_{2}(N)\right)=\left(S_{1}\left(H_{N}\right), S_{2}\left(V_{N}\right)\right) .
$$

\subsection{Recurrence, transience}

Our result on recurrence is a simple application of the celebrated Nash-Williams theorem [21]. To state this result we need some definitions. Consider a Markov chain $(\mathbf{X}, \mathbf{Y}, p)$ with countable state space $\mathbf{X}$, process $\mathbf{Y}$ and transition probabilities $p(\mathbf{u}, \mathbf{v})$. The chain is reversible if there exist strictly positive weights $\pi_{\mathbf{u}}$ for all $\mathbf{u} \in \mathbf{X}$ such that

$$
\pi_{\mathbf{u}} p(\mathbf{u}, \mathbf{v})=\pi_{\mathbf{v}} p(\mathbf{v}, \mathbf{u})
$$


If the chain is reversible we will use

$$
a(\mathbf{u}, \mathbf{v}):=\pi_{\mathbf{u}} p(\mathbf{u}, \mathbf{v})
$$

Obviously the above defined anisotropic walk is a Markov chain on the state space $\mathbf{X}=\mathbb{Z}^{2}$, with the transition probabilities defined in (1.1). Furthermore, this Markov chain is reversible with the strictly positive weights

$$
\pi(k, j)=\frac{1}{p_{j}}
$$

and

$$
\begin{aligned}
& a((k, j),(k, j+1))=a((k, j),(k, j-1))=1 \\
& a((k, j),(k+1, j))=a((k, j),(k-1, j))=\frac{1}{2 p_{j}}-1
\end{aligned}
$$

(and for non nearest neighbor sites $a(.,)=$.0 ). This Markov chain is also time homogeneous, irreducible, i.e. it is possible to get to any state from any state with positive probability. The invariant measure is given by

$$
\mu(k, j)=\pi(k, j)=\frac{1}{p_{j}}, \quad(k, j) \in \mathbb{Z}^{2},
$$

i.e.,

$$
\mu(\mathbf{u})=\sum_{\mathbf{v}} \mu(\mathbf{v}) p(\mathbf{v}, \mathbf{u})
$$

where the summation goes for the four nearest neighbors of $\mathbf{u}$.

Theorem A (Nash-Williams [21]): Suppose that $(\mathbf{X}, \mathbf{Y}, p)$ is a reversible Markov chain and that $\mathbf{X}=\bigcup_{k=0}^{\infty} \Lambda^{k}$ where $\Lambda^{k}$ are disjoint. Suppose further that $\mathbf{u} \in \Lambda^{k}$ and $a(\mathbf{u}, \mathbf{v})>0$ together imply that $\mathbf{v} \in \Lambda^{k-1} \cup \Lambda^{k} \cup \Lambda^{k+1}$, and that for each $k$ the sum

$\sum_{\Lambda^{k}, \mathbf{v} \in \mathbf{X}} a(\mathbf{u}, \mathbf{v})<\infty$. Let $\left[\Lambda^{k}, \Lambda^{k+1}\right]$ denote the set of pairs $(\mathbf{u}, \mathbf{v})$ such that $\mathbf{u} \in \Lambda^{k}$

and $\mathbf{v} \in \Lambda^{k+1}$. The Markov chain is recurrent if

$$
\sum_{k=0}^{\infty}\left(\sum_{(\mathbf{u}, \mathbf{v}) \in\left[\Lambda^{k}, \Lambda^{k+1}\right]} a(\mathbf{u}, \mathbf{v})\right)^{-1}=\infty .
$$

To apply this theorem, let $\Lambda^{k}$ be the set of $8 k$ lattice points on the square of width $2 k$, centered at the origin. Furthermore, let $\left[\Lambda^{k}, \Lambda^{k+1}\right]$ be the set of $8 k+4$ nearest neighbor pairs (edges) between $\Lambda^{k}$ and $\Lambda^{k+1}$.

It is easy to see by (1.4) that the sum in (1.6) is equal to

$$
\sum_{k=0}^{\infty}\left(2\left(\sum_{j=-k}^{k}\left(\frac{1}{2 p_{j}}-1\right)+\sum_{j=-k}^{k} 1\right)\right)^{-1}=\sum_{k=0}^{\infty}\left(\sum_{j=-k}^{k} \frac{1}{p_{j}}\right)^{-1} .
$$


So we got the following result.

Theorem 1.1 The anisotropic walk is recurrent if

$$
\sum_{k=0}^{\infty}\left(\sum_{j=-k}^{k} \frac{1}{p_{j}}\right)^{-1}=\infty
$$

As a simple consequence, if $\min _{j \in \mathbb{Z}} p_{j}>0$, then the anisotropic walk is recurrent.

It is an intriguing question whether the converse of this statement is true as well. That is to say, is it true that

\section{Conjecture 1.1 If}

$$
\sum_{k=0}^{\infty}\left(\sum_{j=-k}^{k} \frac{1}{p_{j}}\right)^{-1}<\infty
$$

then the anisotropic walk is transient.

We can't prove this conjecture, but a somewhat weaker result is true.

Theorem 1.2 Assume that

$$
\sum_{j=-k}^{k} \frac{1}{p_{j}}=C k^{1+A}+O\left(k^{1+A-\delta}\right), \quad k \rightarrow \infty
$$

for some $C>0, A>0$ and $0<\delta \leq 1$. Then the anisotropic random walk is transient.

Proof. Consider the simple symmetric random walk $S_{2}(\cdot)$ of the vertical steps and let $\xi_{2}(\cdot, \cdot)$ be its local time, and $\rho_{2}(\cdot)$ be its return time to zero. Consider the anisotropic random walk of $N$ steps, where $N=N(m)$ is the time of $m$-th return of $S_{2}(\cdot)$ to zero, i.e., let $V_{N}=\rho_{2}(m)$.

First we give a lower bound for the number of the horizontal steps $H_{N}$.

Lemma 1. For small enough $\varepsilon>0$ we have almost surely for large enough $m$

$$
H_{N}=H_{N(m)} \geq m^{1+(1-\varepsilon)(A+1)} .
$$

Proof. For simplicity in the proof, we denote $\xi_{2}$ by $\xi$ and $\rho_{2}$ by $\rho$. From the construction in Section 1.1 it can be seen that the number of horizontal steps up to the $m$-th return to zero by the vertical component is given by

$$
H_{N}=\sum_{j=-\infty}^{\infty} \sum_{i=1}^{\xi(j, \rho(m))} G_{i}^{(j)}
$$

where $G_{i}^{j}$ are as in Section 1.1. Since $\rho(m) \geq m^{2-\varepsilon}$ for small $\varepsilon>0$ and large enough $m$ almost surely, it follows from the stability of local time (see [23], Theorem 11.22, p. 127), that for any $\varepsilon>0,|j| \leq m^{1-\varepsilon}$ we have 


$$
(1-\varepsilon) m \leq \xi(j, \rho(m)) \leq(1+\varepsilon) m
$$

almost surely for large enough $m$. Hence

$$
H_{N} \geq \sum_{|j| \leq m^{1-\varepsilon}} \sum_{i=1}^{(1-\varepsilon) m} G_{i}^{(j)}=: U_{m}
$$

We consider the expectation of $U_{m}$ and show that the other terms are negligible. We have

$$
\begin{gathered}
\mathbf{E} G_{i}^{(j)}=\frac{1-2 p_{j}}{2 p_{j}}, \\
\operatorname{VarG}_{i}^{(j)}=\frac{1-2 p_{j}}{4 p_{j}^{2}} .
\end{gathered}
$$

Hence by (1.9) we get

$$
\mathbf{E} U_{m}=m(1-\varepsilon) \sum_{|j| \leq m^{1-\varepsilon}} \frac{1-2 p_{j}}{2 p_{j}} \geq c m^{1+(1-\varepsilon)(A+1)},
$$

where $c>0$ is a constant. In what follows the value of such a $c$ might change from line to line. We have

$$
\operatorname{Var}_{m}=m(1-\varepsilon) \sum_{|j| \leq m^{1-\varepsilon}} \frac{1-2 p_{j}}{4 p_{j}^{2}} .
$$

It follows from (1.9) that $\frac{1}{2 p_{j}} \leq c|j|^{1+A-\delta}$, hence

$$
\operatorname{Var} U_{m} \leq c m(1-\varepsilon) \sum_{|j| \leq m^{1-\varepsilon}} \frac{|j|^{1+A-\delta}}{p_{j}} \leq c m^{1+(1-\varepsilon)(2+2 A-\delta)} .
$$

By Chebyshev inequality

$$
\mathbf{P}\left(\left|U_{m}-\mathbf{E} U_{m}\right| \geq m^{(1-\varepsilon)(A+2)}\right) \leq c \frac{m^{3+2 A-2 \varepsilon(1+A)-(1-\varepsilon) \delta}}{m^{2(1-\varepsilon)(A+2)}}=c m^{-1-(1-\varepsilon) \delta+2 \varepsilon}
$$

which, by choosing $\varepsilon$ small enough, is summable. Hence, as $m \rightarrow \infty$,

$$
U_{m}=\mathbf{E} U_{m}+O\left(m^{(1-\varepsilon)(A+2)}\right) \quad \text { a.s. }
$$

consequently

$$
H_{N} \geq U_{m} \geq c m^{1+(1-\varepsilon)(1+A)}
$$

almost surely for large $m$.

Lemma 2. Let $S(\cdot)$ be a simple symmetric random walk and let $r(m)$ be a sequence of integer valued random variables independent of $S(\cdot)$ and such that $r(m) \geq m^{\beta}$ almost surely for large $m$ with $\beta>2$. Then with small enough $\varepsilon>0$ we have 


$$
|S(r(m))| \geq m^{\beta / 2-1-\varepsilon}
$$

almost surely for large $m$.

Proof. From the local central limit theorem

$$
\mathbf{P}(S(k)=j) \leq \frac{c}{\sqrt{k}}
$$

for all $k \geq 0$ and $j \in \mathbb{Z}$ with an absolute constant $c>0$. Hence

$$
\mathbf{P}\left(\frac{|S(k)|}{\sqrt{k}} \leq x\right)=\sum_{|j| \leq x \sqrt{k}} P(S(k)=j) \leq c x,
$$

This remains true if $k$ is replaced by a random variable, independent of $S(\cdot)$, e.g. $k=r(m)$, i.e. we have

$$
\mathbf{P}\left(\frac{|S(r(m))|}{\sqrt{r(m)}} \leq \frac{1}{m^{1+\varepsilon}}\right) \leq c \frac{1}{m^{1+\varepsilon}},
$$

consequently by Borel-Cantelli Lemma

$$
|S(r(m))| \geq \frac{\sqrt{r(m)}}{m^{1+\varepsilon}} \geq m^{\beta / 2-1-\varepsilon},
$$

almost surely for all large enough $m$. This completes the proof of the Lemma.

Applying the two lemmas with $r(m)=H_{N(m)}$, we get

$$
\left|S_{1}\left(H_{N}\right)\right| \geq m^{A / 2-\varepsilon A / 2-3 \varepsilon / 2}=m^{\gamma}
$$

with $\gamma>0$ by choosing $\varepsilon>0$ small enough. It follows that for large $N, S_{1}\left(H_{N}\right)$ almost surely can't be equal to zero.

Let

$$
A_{m}:=\left\{\exists j, \rho_{2}(m)<j<\rho_{2}(m+1) \quad \text { such that } \quad \mathbf{C}(j)=(0,0)\right\} .
$$

Clearly $A_{m}$ could only happen if from $\rho_{2}(m)$ to $\rho_{2}(m+1)$ the walk only steps horizontally (if it makes one vertical step the return to the origin could only happen after or at $\left.\rho_{2}(m+1)\right)$. Thus by our lemmas in order that $A_{m}$ could happen, the walk needs to have at least $m^{\gamma}$ consecutive steps on the $x$-axis, thus

$$
\sum_{m}^{\infty} \mathbf{P}\left(A_{m}\right) \leq \sum_{m}^{\infty}\left(1 / 2-p_{0}\right)^{m^{\gamma}}<\infty .
$$

So the anisotropic random walk cannot return to zero infinitely often with probability 1 , it is transient. This proves the theorem. 


\section{Strong approximations}

In this section we present results concerning strong approximations of the twodimensional anisotropic random walks. Of course, the results are different in the various cases, and in some cases the problem is open. We also mention weak convergence results available in the literature. First we describe the general method how to obtain these strong approximations.

Assume that our anisotropic random walk is constructed on a probability space as described in Section 1.1, and in accordance with Theorems 6.1 and 10.1 of Révész [23] we may assume that on the same probability space there are also two independent standard Wiener processes (Brownian motions) $W_{i}(\cdot), i=1,2$ with local times $\eta_{i}(\cdot, \cdot)$ such that for all $\varepsilon>0$, as $n \rightarrow \infty$, we have

$$
S_{i}(n)=W_{i}(n)+O\left(n^{1 / 4+\varepsilon}\right) \quad \text { a.s. }
$$

and

$$
\xi_{i}(j, n)=\eta_{i}(j, n)+O\left(n^{1 / 4+\varepsilon}\right) \quad \text { a.s. }
$$

Then

$$
C_{1}(N)=S_{1}\left(H_{N}\right)=W_{1}\left(H_{N}\right)+O\left(H_{N}^{1 / 4+\varepsilon}\right) \quad \text { a.s. }
$$

and

$$
C_{2}(N)=S_{2}\left(V_{N}\right)=W_{2}\left(V_{N}\right)+O\left(V_{N}^{1 / 4+\varepsilon}\right) \quad \text { a.s. },
$$

if $H_{N} \rightarrow \infty$ and $V_{N} \rightarrow \infty$ as $N \rightarrow \infty$, almost surely.

So we have to give reasonable approximations to $H_{N}$ and $V_{N}$, or at least to one of them, and use $V_{N}+H_{N}=N$.

It turned out that in many cases treated, the following is a good approximation of $H_{N}$.

$$
H_{N} \sim \sum_{j} \sum_{i=1}^{\xi_{2}(j, n)} G_{i}^{(j)} \sim \sum_{j} \xi_{2}(j, n) \frac{1-2 p_{j}}{2 p_{j}}
$$

with $n=V_{N} . H_{N}$ and the double sum above are not necessarily equal, since the last geometric variable might be truncated in $H_{N}$. So we have to investigate the additive functional

$$
A(n)=\sum_{j} f(j) \xi_{2}(j, n)=\sum_{k=0}^{n} f\left(S_{2}(k)\right), \quad f(j)=\frac{1-2 p_{j}}{2 p_{j}}
$$

of the vertical component and approximate it by the additive functional of $W_{2}(\cdot)$

$$
B(t)=\int_{-\infty}^{\infty} f(x) \eta_{2}(x, t) d x=\int_{0}^{t} f\left(W_{2}(s)\right) d s
$$

where between integers we define $f(x)=f(j), j \leq x<j+1$.

In certain cases the following Lemma, equivalent to Lemma 2.3 of Horváth [17], giving strong approximation of additive functionals, may be useful. 
Lemma A Let $g(t)$ be a non-negative function such that $g(t)=g(j), j \leq t<j+1$, for $j \in \mathbb{Z}$ and assume that $g(t) \leq c\left(|t|^{\beta}+1\right)$ for some $0<c$ and $\beta \geq 0$. Then

$$
\left|\sum_{j=0}^{n} g\left(S_{2}(j)\right)-\int_{0}^{n} g\left(W_{2}(s)\right) d s\right|=o\left(n^{\beta / 2+3 / 4+\varepsilon}\right) \quad \text { a.s. }
$$

as $n \rightarrow \infty$.

Now let us introduce the notations

$$
\sum_{j=1}^{k} f(j)=b_{k}, \quad \sum_{j=1}^{k} f(-j)=c_{k}
$$

The next assumption is a reasonable one used in the literature: as $k \rightarrow \infty$

$$
\begin{aligned}
& b_{k}=(\gamma-1) k^{\alpha}+o\left(k^{\alpha-\delta}\right) \\
& c_{k}=(\gamma-1) k^{\alpha}+o\left(k^{\alpha-\delta}\right)
\end{aligned}
$$

with some $\gamma \geq 1, \alpha \geq 0$ and $\delta>0$. Observe that (1.2) is a particular case with $\alpha=1$.

We consider the following cases:

(1) $\alpha=0$

(2) $0<\alpha<1$

(3) $\alpha=1$

(4) $\alpha>1$

(5) nonsymmetric case, i.e. the constants $\gamma$ in (2.1) and (2.2) are different.

\subsection{The case $\alpha=0$}

The most interesting and well established case is the so-called comb structure, i.e., $p_{0}=1 / 4, p_{j}=1 / 2, j= \pm 1, \pm 2, \ldots$. It follows from Theorem 1.1 that the random walk in this case is recurrent. We note in passing the interesting result of KrishnapurPeres [19]: two independent random walks on the comb meet only finitely often with probability 1 .

For random walk on comb we refer to Weiss and Havlin [29], Bertacchi and Zucca [2] and references given there. The following result on weak convergence was established by Bertacchi [1].

\section{Theorem B}

$$
\left(\frac{C_{1}(N t)}{N^{1 / 4}}, \frac{C_{2}(N t)}{N^{1 / 2}} ; t \geq 0\right) \stackrel{\text { Law }}{\longrightarrow}\left(W_{1}\left(\eta_{2}(0, t)\right), W_{2}(t) ; t \geq 0\right), \quad N \rightarrow \infty .
$$

Strong approximation was given in Csáki et al. [5].

Theorem 2.1 On an appropriate probability space we have 


$$
N^{-1 / 4}\left|C_{1}(N)-W_{1}\left(\eta_{2}(0, N)\right)\right|+N^{-1 / 2}\left|C_{2}(N)-W_{2}(N)\right|=O\left(N^{-1 / 8+\varepsilon}\right) \quad \text { a.s. },
$$

as $N \rightarrow \infty$.

We have the following consequences.

\section{Corollary 1.}

$$
\begin{gathered}
\limsup _{N \rightarrow \infty} \frac{C_{1}(N)}{2^{5 / 4} 3^{-3 / 4} N^{1 / 4}(\log \log N)^{3 / 4}}=1 \quad \text { a.s. } \\
\limsup _{N \rightarrow \infty} \frac{C_{2}(N)}{(2 N \log \log N)^{1 / 2}}=1 \quad \text { a.s. }
\end{gathered}
$$

For general results in the case $\alpha=0$ we just remark that in this case $\bar{f}=\sum_{j} f(j)$ is convergent, then by our assumptions its terms are non-negative and at least one of them is strictly positive, hence $\bar{f}>0$. By the ratio ergodic theorem (cf., e.g., Theorem 3.6 in Revuz [24])

$$
A(n) \sim \bar{f} \xi_{2}(0, n), \quad \bar{f}=\sum_{j} f(j)=2(\gamma-1)+f(0)
$$

almost surely, as $n \rightarrow \infty$, hence

$$
A(n)=O\left((n \log \log n)^{1 / 2}\right) \quad \text { a.s., } n \rightarrow \infty .
$$

Let

$$
H_{N}^{+}=\sum_{j} \sum_{i=1}^{\xi_{2}(j, n)} G_{i}^{(j)}, \quad H_{N}^{-}=\sum_{j} \sum_{i=1}^{\xi_{2}(j, n)-1} G_{i}^{(j)} .
$$

Obviously, $H_{N}^{-} \leq H_{N} \leq H_{N}^{+}$. Consider $H_{N}^{+}$, which is a (random) sum of independent random variables. Under the condition $\mathscr{F}=\left\{S_{2}(k), k \geq 0\right\}$ we have

$$
\begin{gathered}
E\left(H_{N}^{+} \mid \mathscr{F}\right)=\sum_{j} f(j) \xi_{2}(j, n)=A(n) \\
\operatorname{Var}\left(H_{N}^{+} \mid \mathscr{F}\right)=\sum_{j} \frac{f(j)}{2 p_{j}} \xi_{2}(j, n) .
\end{gathered}
$$

It is easy to see that the $\operatorname{sum} \sum_{j} f(j) / 2 p_{j}$ is also convergent, hence

$$
\operatorname{Var}\left(H_{N}^{+} \mid \mathscr{F}\right) \sim c \xi(0, n)
$$

with some $c>0$. Now apply Theorem 6.17 in Petrov [22] saying that for sums of independent (not necessary identically distributed) random variables we have

$$
\sum_{i} X_{i}=\sum_{i} E X_{i}+o\left(\left(\sum_{i} \operatorname{Var} X_{i}\right)^{1 / 2+\varepsilon}\right)
$$


almost surely. Thus

$$
H_{N}^{+}=\bar{f} \xi_{2}(0, n)(1+o(1))=\bar{f} \xi_{2}\left(0, V_{N}\right)(1+o(1))
$$

almost surely as $N \rightarrow \infty$. Similar results are true for $H_{N}^{-}$, hence also for $H_{N}$, i.e.

$$
H_{N}=\bar{f} \xi_{2}(0, n)(1+o(1))=\bar{f} \xi_{2}\left(0, V_{N}\right)(1+o(1)) .
$$

Since $C_{1}(N)=S_{1}\left(H_{N}\right)$, using that $H_{N}=O\left((N \log \log N)^{1 / 2}\right)$ and the strong approximations of $S_{1}(\cdot), S_{2}(\cdot)$ by $W_{1}(\cdot), W_{2}(\cdot)$ and $\xi_{2}(0, \cdot)$ by $\eta_{2}(\cdot)$, we can obtain the following limit distribution: as $N \rightarrow \infty$,

$$
\left(\frac{C_{1}(N)}{N^{1 / 4}}, \frac{C_{2}(N)}{N^{1 / 2}}\right) \stackrel{d}{\rightarrow}\left(W_{1}\left(\bar{f} \eta_{2}(0,1)\right), W_{2}(1)\right) .
$$

Further results, like strong approximations, remain to be established in this case.

\subsection{The case $0<\alpha<1$}

This is also a recurrent case, but approximations, limit theorems, etc. remain to be worked out in detail. We just note that from the law of the iterated logarithm for the local time we have

$$
A(n)=\sum_{j} f(j) \xi_{2}(j, n) \leq c(n \log \log n)^{(1+\alpha) / 2},
$$

a.s., $n \rightarrow \infty$, hence the vertical part dominates, i.e., as $N \rightarrow \infty$ we should have

$$
H_{N}=O\left((N \log \log N)^{(1+\alpha) / 2}\right)<<N \quad \text { a.s. },
$$

and we expect that

$$
C_{1}(N)=W_{1}(Z(N))+O\left(N^{(1+\alpha) / 4+\varepsilon}\right) \quad \text { a.s. },
$$

where

$$
Z(N)=\int_{0}^{N} f\left(W_{2}(s)\right) d s,
$$

and for the vertical component

$$
C_{2}(N)=W_{2}(N)+O\left(N^{1 / 2-\varepsilon}\right), \quad \text { a.s. }
$$

as $N \rightarrow \infty$. 


\subsection{The case $\alpha=1$}

Assume also that $\delta>1 / 2, \gamma>1$.

It can be seen from Thoerem 1.1 that the anisotropic random walk in this case is recurrent.

Heyde [14] gave the following strong approximation:

Theorem C On an appropriate probability space we have

$$
\gamma^{1 / 2} C_{2}(N)=W_{2}\left(N\left(1+\varepsilon_{N}\right)\right)+O\left(N^{1 / 4}(\log N)^{1 / 2}(\log \log N)^{1 / 2}\right) \quad \text { a.s. }
$$

as $N \rightarrow \infty$, where $W(\cdot)$ is a standard Wiener process and $\lim _{N \rightarrow \infty} \varepsilon_{N}=0$ a.s.

In another paper Heyde [15] gave weak convergence result for both coordinates.

\section{Theorem D}

$$
\left(\frac{C_{1}(N)}{N^{1 / 2}}, \frac{C_{2}(N)}{N^{1 / 2}}\right) \stackrel{d}{\rightarrow}\left(W_{1}\left(1-\gamma^{-1}\right), W_{2}\left(\gamma^{-1}\right)\right) .
$$

Strong approximation result for both coordinates was given in Csáki et al. [9]:

Theorem 2.2 On an appropriate probability space we have for any $\varepsilon>0$

$$
\left|C_{1}(N)-W_{1}\left(\frac{(\gamma-1) N}{\gamma}\right)\right|+\left|C_{2}(N)-W_{2}\left(\frac{N}{\gamma}\right)\right|=O\left(N^{5 / 8-\delta / 4+\varepsilon}\right) \quad \text { a.s. }
$$

as $N \rightarrow \infty$.

Moreover, in the periodic case, when $p_{j}=p_{j+L}$ for each $j \in \mathbb{Z}$ and a fixed integer $L \geq 1$ we have

$$
\left|C_{1}(N)-W_{1}\left(\frac{(\gamma-1) N}{\gamma}\right)\right|+\left|C_{2}(N)-W_{2}\left(\frac{N}{\gamma}\right)\right|=O\left(N^{1 / 4+\varepsilon}\right) \quad \text { a.s. }
$$

as $N \rightarrow \infty$, where

$$
\gamma=\frac{\sum_{j=0}^{L-1} p_{j}^{-1}}{2 L}
$$

Some consequences are the following laws of the iterated logarithm.

$$
\begin{gathered}
\limsup _{N \rightarrow \infty} \frac{C_{1}(N)}{(N \log \log N)^{1 / 2}}=\left(\frac{2(\gamma-1)}{\gamma}\right)^{1 / 2} \quad \text { a.s. } \\
\limsup _{N \rightarrow \infty} \frac{C_{2}(N)}{(N \log \log N)^{1 / 2}}=\left(\frac{2}{\gamma}\right)^{1 / 2} \text { a.s. }
\end{gathered}
$$

\subsection{The case $\alpha>1$}

In this case the random walk is transient by Theorem 1.2. 
It is an open problem to give strong approximations in this case. Horváth [17] established weak convergence of $C_{2}(\cdot)$ to some time changed Wiener process. We mention a particular case of his results, valid for all $\alpha>1$.

Let

$$
I_{\alpha}(t)=\int_{0}^{t}\left|W_{2}(s)\right|^{\alpha-1} d s
$$

$I_{\alpha}$ is strictly increasing, so we can define its inverse, denoted by $J_{\alpha}$. Then we have

$$
\frac{C_{2}(N t)}{N^{1 /(1+\alpha)}} \stackrel{\text { Law }}{\longrightarrow} c_{0} W_{2}\left(J_{\alpha}(t)\right)
$$

with some constant $c_{0}$.

In this case the number of horizontal steps dominates the number of vertical steps, therefore $C_{1}(N)$ might be approximated by $W_{1}(N)$.

\subsection{Unsymmetric case}

Some weak convergence in this case was treated in Heyde et al. [16] and Horváth [17]. Strong approximation in a particular case, the so-called half-plane half-comb structure (HPHC) was given in Csáki et al. [8].

Let $p_{j}=1 / 4, j=0,1,2, \ldots$ and $p_{j}=1 / 2, j=-1,-2, \ldots$, i.e., a square lattice on the upper half-plane, and a comb structure on the lower half plane. Let furthermore

$$
\alpha_{2}(t):=\int_{0}^{t} I\left\{W_{2}(s) \geq 0\right\} d s
$$

i.e., the time spent by $W_{2}$ on the non-negative side during the interval $[0, t]$. The process $\gamma_{2}(t):=\alpha_{2}(t)+t$ is strictly increasing, hence we can define its inverse: $\beta_{2}(t):=\left(\gamma_{2}(t)\right)^{-1}$.

Theorem 2.3 On an appropriate probability space we have

$$
\left|C_{1}(N)-W_{1}\left(N-\beta_{2}(N)\right)\right|+\left|C_{2}(N)-W_{2}\left(\beta_{2}(N)\right)\right|=O\left(N^{3 / 8+\varepsilon}\right) \quad \text { a.s. }
$$

as $N \rightarrow \infty$.

The following laws of the iterated logarithm hold:

\section{Corollary 2.}

$$
\begin{gathered}
\limsup _{t \rightarrow \infty} \frac{W_{1}\left(t-\beta_{2}(t)\right)}{\sqrt{t \log \log t}}=\limsup _{N \rightarrow \infty} \frac{C_{1}(N)}{\sqrt{N \log \log N}}=1 \quad \text { a.s. } \\
\liminf _{t \rightarrow \infty} \frac{W_{1}\left(t-\beta_{2}(t)\right)}{\sqrt{t \log \log t}}=\liminf _{N \rightarrow \infty} \frac{C_{1}(N)}{\sqrt{N \log \log N}}=-1 \quad \text { a.s. } \\
\limsup _{t \rightarrow \infty} \frac{W_{2}\left(\beta_{2}(t)\right)}{\sqrt{t \log \log t}}=\limsup _{N \rightarrow \infty} \frac{C_{2}(N)}{\sqrt{N \log \log N}}=1 \quad \text { a.s. }
\end{gathered}
$$




$$
\liminf _{t \rightarrow \infty} \frac{W_{2}\left(\beta_{2}(t)\right)}{\sqrt{t \log \log t}}=\liminf _{N \rightarrow \infty} \frac{C_{2}(N)}{\sqrt{N \log \log N}}=-\sqrt{2} \quad \text { a.s. }
$$

\section{Local time}

We don't know any general result about the local time of the anisotropic walk. It would require to determine asymptotic results or at least good estimations for the return probabilities, i.e., we would need local limit theorems. In fact, we know such results in two cases: the periodic and the comb structure case.

\subsection{Periodic anisotropic walk}

In case of the periodic anisotropic walk, i.e., when $p_{j}=p_{j+L}$, for some fixed integer $L \geq 1$ and $j=0, \pm 1, \pm 2, \ldots$ we know the following local limit theorem for the random walk denoted by $\mathbf{C}^{\mathbf{P}}(\cdot)$.

Lemma 1. As $N \rightarrow \infty$, we have

$$
\mathbf{P}\left(\mathbf{C}^{\mathbf{P}}(2 N)=(0,0)\right) \sim \frac{1}{4 \pi N p_{0} \sqrt{\gamma-1}}
$$

with $\gamma=\sum_{j=0}^{L-1} p_{j}^{-1} /(2 L)$.

The proof of this lemma is based on the work of Roerdink and Shuler [25]. It follows from this lemma, that the truncated Green function $g(\cdot)$ is given by

$$
g(N)=\sum_{k=0}^{N} \mathbf{P}\left(\mathbf{C}^{\mathbf{P}}(k)=(0,0)\right) \sim \frac{\log N}{4 p_{0} \pi \sqrt{\gamma-1}}, \quad N \rightarrow \infty,
$$

which implies that our anisotropic random walk in this case is recurrent and also Harris recurrent.

First, we define the local time by

$$
\Xi((k, j), N)=\sum_{r=1}^{N} I\left\{\mathbf{C}^{\mathbf{P}}(r)=(k, j)\right\}, \quad(k, j) \in \mathbb{Z}^{2} .
$$

In the case when the random walk is (Harris) recurrent, then we have (cf. e.g. Chen [4])

$$
\lim _{N \rightarrow \infty} \frac{\Xi\left(\left(k_{1}, j_{1}\right), N\right)}{\Xi\left(\left(k_{2}, j_{2}\right), N\right)}=\frac{\mu\left(k_{1}, j_{1}\right)}{\mu\left(k_{2}, j_{2}\right)} \quad \text { a.s. }
$$

where $\mu(\cdot)$ is an invariant measure. Hence by (1.5) 


$$
\lim _{N \rightarrow \infty} \frac{\Xi((0,0), N)}{\Xi((k, j), N)}=\frac{p_{j}}{p_{0}} \quad \text { a.s. }
$$

for $(k, j) \in \mathbb{Z}^{2}$ fixed.

Thus, using now $g(N)$, it follows from Darling and Kac [11] that we have

\section{Corollary 3.}

$$
\lim _{N \rightarrow \infty} \mathbf{P}\left(\frac{\Xi((0,0), N)}{g(N)} \geq x\right)=\lim _{N \rightarrow \infty} \mathbf{P}\left(\frac{4 p_{0} \pi \sqrt{\gamma-1} \Xi((0,0), N)}{\log N} \geq x\right)=e^{-x}
$$

for $x \geq 0$.

For a limsup result, via Chen [4] we conclude

\section{Corollary 4.}

$$
\limsup _{N \rightarrow \infty} \frac{\Xi((0,0), N)}{\log N \log \log \log N}=\frac{1}{4 p_{0} \pi \sqrt{\gamma-1}} \quad \text { a.s. }
$$

\subsection{Comb}

Now we consider the case of the two-dimensional comb structure $\mathbb{C}^{2}$, i.e., when $p_{0}=1 / 4$ and $p_{j}=1 / 2$ for $j= \pm 1, \pm 2, \ldots$

First we give the return probability from Woess [31], p. 197:

$$
\mathbf{P}(\mathbf{C}(2 N)=(0,0)) \sim \frac{2^{1 / 2}}{\Gamma(1 / 4) N^{3 / 4}}, \quad N \rightarrow \infty .
$$

This result indicates that the local time tipically is of order $N^{1 / 4}$. In Csáki et al. [6] and [7] we have shown the following results.

Theorem 3.1 The limiting distribution of the local time is given by

$$
\lim _{N \rightarrow \infty} \mathbf{P}\left(\Xi((0,0), N) / N^{1 / 4}<x\right)=\mathbf{P}\left(2 \eta_{1}\left(0, \eta_{2}(0,1)\right)<x\right)=\mathbf{P}(2|U| \sqrt{|V|}<x),
$$

where $U$ and $V$ are two independent standard normal random variables.

Concerning strong approximation, in Csáki et al. [7] we proved the following results.

Theorem 3.2 On an appropriate probability space for the random walk $\left\{\mathbf{C}(N)=\left(C_{1}(N), C_{2}(N)\right) ; N=0,1,2, \ldots\right\}$ on $\mathbb{C}^{2}$, one can construct two independent standard Wiener processes $\left\{W_{1}(t) ; t \geq 0\right\},\left\{W_{2}(t) ; t \geq 0\right\}$ with their corresponding local time processes $\eta_{1}(\cdot, \cdot), \eta_{2}(\cdot, \cdot)$ such that, as $N \rightarrow \infty$, we have for any $\delta>0$

$$
\sup _{x \in \mathbb{Z}}\left|\Xi((x, 0), N)-2 \eta_{1}\left(x, \eta_{2}(0, N)\right)\right|=O\left(N^{1 / 8+\delta}\right) \quad \text { a.s. }
$$


The next result shows that on the backbone up to $|x| \leq N^{1 / 4-\varepsilon}$ we have uniformity, all the sites have approximately the same local time as the origin. Furthermore if we consider a site on a tooth of the comb its local time is roughly half of the local time of the origin. This is pretty natural, as it turns out from the proof that on the backbone the number of horizontal and vertical visits to any particular site are approximately equal.

Theorem 3.3 On the probability space of Theorem 3.2, as $N \rightarrow \infty$, we have for any $0<\varepsilon<1 / 4$

$$
\max _{|x| \leq N^{1 / 4-\varepsilon}}|\Xi((x, 0), N)-\Xi((0,0), N)|=O\left(N^{1 / 4-\delta}\right) \quad \text { a.s. }
$$

and

$$
\max _{0<|y| \leq N^{1 / 4-\varepsilon}} \max _{|x| \leq N^{1 / 4-\varepsilon}}\left|\Xi((x, y), N)-\frac{1}{2} \Xi((0,0), N)\right|=O\left(N^{1 / 4-\delta}\right) \quad \text { a.s. },
$$

for any $0<\delta<\varepsilon / 2$, where the maximum is taken on the integers.

It would be an interesting problem to investigate the local time for $|y|>N^{1 / 4}$. We believe e.g. that the maximal local time taken for all $(x, y) \in \mathbb{Z}^{2}$ is of order $N^{1 / 2}$. Such results however remain to be established.

One of our old results [10] describes the Strassen class of $\eta_{1}\left(0, \eta_{2}(0, z t)\right)$ as follows. This, combined with Theorems 3.2 and 3.3, allows us to conclude the corresponding Strassen class result for the local times of the walk.

Theorem 3.4 The net

$$
\left\{\frac{\eta_{1}\left(0, \eta_{2}(0, z t)\right)}{2^{5 / 4} 3^{-3 / 4} t^{1 / 4}(\log \log t)^{3 / 4}} ; 0 \leq z \leq 1\right\}_{t \geq 3}
$$

as $t \rightarrow \infty$, is almost surely relatively compact in the space $C([0,1], \mathbb{R})$ of continuous functions from $[0,1]$ to $\mathbb{R}$, and the set of its limit points is the class of nondecreasing absolutely continuous functions (with respect to the Lebesgue measure) on $[0,1]$ for which

$$
\mathscr{S}^{*}:\left\{f(0)=0 \text { and } \int_{0}^{1}|\dot{f}(x)|^{4 / 3} d x \leq 1\right\} .
$$

Some obvious consequences of these results are the following

- $\limsup _{t \rightarrow \infty} \frac{\eta_{1}\left(0, \eta_{2}(0, t)\right)}{t^{1 / 4}(\log \log t)^{3 / 4}}=\frac{2^{5 / 4}}{3^{3 / 4}} \quad$ a.s.

- $\limsup _{N \rightarrow \infty} \frac{\Xi((x, 0), N)}{N^{1 / 4}(\log \log N)^{3 / 4}}=\frac{2^{9 / 4}}{3^{3 / 4}}$ a.s.,

- $\limsup _{N \rightarrow \infty} \frac{\Xi((x, y), N)}{N^{1 / 4}(\log \log N)^{3 / 4}}=\frac{2^{5 / 4}}{3^{3 / 4}} \quad$ a.s. $y \neq 0$.

A beautiful classical result of Lévy, P. [18] reads as follows 
Theorem $\mathbf{E}$ Let $W(\cdot)$ be a standard Wiener process with local time process $\eta(\cdot, \cdot)$. The following equality in distribution holds:

$$
\{\eta(0, t), t \geq 0\} \stackrel{d}{=}\left\{\sup _{0 \leq s \leq t} W(s), t \geq 0\right\} .
$$

Consequently using a Hirsch type result of Bertoin [3], we get

Corollary 5. Let $\beta(t)>0, t \geq 0$, be a non-increasing function. Then we have almost surely that

$$
\liminf _{t \rightarrow \infty} \frac{\eta_{1}\left(0, \eta_{2}(0, t)\right)}{t^{1 / 4} \beta(t)}=0 \quad \text { or } \quad \infty
$$

according as the integral $\int_{1}^{\infty} \beta(t) / t d t$ diverges or converges.

So we also have

Corollary 6. Let $\beta(n), n=1,2, \ldots$ be a non-increasing sequence of positive numbers. Then, for any fixed $(x, y) \in \mathbb{Z}^{2}$, we have almost surely that

$$
\liminf _{n \rightarrow \infty} \frac{\Xi((x, y), n)}{n^{1 / 4} \beta(n)}=0 \quad \text { or } \quad \infty
$$

according as the series $\sum_{1}^{\infty} \beta(n) / n$ diverges or converges.

Now we also might consider the behavior of the supremum of the local time over the backbone. To this end we first had to prove the following pair of integral tests for the $\sup _{x \in \mathbb{R}} \eta_{1}\left(x, \eta_{2}(0, t)\right)$ process.

Theorem 3.5 Let $f(t)>0$ be a non-decreasing function and put

$$
I(f):=\int_{1}^{\infty} \frac{f^{2}(t)}{t} \exp \left(-\frac{3}{2^{5 / 3}} f^{4 / 3}(t)\right) d t .
$$

Then, as $t \rightarrow \infty$,

$$
\mathbf{P}\left(\sup _{x \in \mathbb{R}} \eta_{1}\left(x, \eta_{2}(0, t)\right)>t^{1 / 4} f(t) \text { i.o. }\right)=0 \text { or } 1
$$

according as $I(f)$ converges or diverges.

Theorem 3.6 Let $g(t)>0$ be a non-increasing function and

$$
J(g):=\int_{1}^{\infty} \frac{g^{2}(t)}{t} d t
$$

Then, as $t \rightarrow \infty$,

$$
\mathbf{P}\left(\sup _{x \in \mathbb{R}} \eta_{1}\left(x, \eta_{2}(0, t)\right)<t^{1 / 4} g(t) \text { i.o. }\right)=0 \text { or } 1
$$

according as whether $J(g)$ converges or diverges. 
The above theorems imply the following integral tests for $\sup _{x \in \mathbb{Z}} \Xi((x, 0), n)$;

Theorem 3.7 Let a $(n)$ be a non-decreasing sequence. Then, as $n \rightarrow \infty$,

$$
\mathbf{P}\left(\sup _{x \in \mathbb{Z}} \Xi((x, 0), n)>2 n^{1 / 4} a(n) \text { i.o. }\right)=0 \text { or } 1
$$

according as

$$
\sum_{n=1}^{\infty} \frac{a^{2}(n)}{n} \exp \left(-\frac{3 a^{4 / 3}(n)}{2^{5 / 3}}\right)<\infty \text { or }=\infty .
$$

Theorem 3.8 Let $b(n)$ be a non-increasing sequence. Then, as $n \rightarrow \infty$,

$$
\mathbf{P}\left(\sup _{x \in \mathbb{Z}} \Xi((x, 0), n)<n^{1 / 4} b(n) \text { i.o. }\right)=0 \text { or } 1
$$

according as

$$
\sum_{n=1}^{\infty} \frac{b^{2}(n)}{n}<\infty \text { or }=\infty
$$

\section{Range}

The range of the anisotropic walk is defined in the usual way as

$$
R(N)=\sum_{(k, j) \in \mathbb{Z}^{2}} I(\Xi((k, j), N)>0)
$$

i.e., the number of distinct sites visited by the random walk up to time $N$, where $\Xi((k, j), N)$ is the local time of the point $(k, j)$ at time $N$.

We are not aware of any all embracing result about the range of the anisotropic walk in general. However the case of the periodic walk is completely understood.

Recall that the walk is periodic if $p_{j}=p_{j+L}$ for each $j \in \mathbb{Z}$, where $L \geq 1$ is a positive integer. In this case it is easy to see that

$$
\gamma=\frac{\sum_{j=0}^{L-1} p_{j}^{-1}}{2 L} .
$$

Roerdink and Shuler [25] gives the asymptotic expected value of the range:

$$
\mathbf{E}(R(N)) \sim \frac{2 \pi \sqrt{\gamma-1}}{\gamma} \frac{N}{\log N}, \quad N \rightarrow \infty .
$$

Moreover, it can be seen that our walk in this case is equivalent to the so-called random walk with internal states, consequently, a law of large numbers follows from Nándori [20] 


$$
\lim _{N \rightarrow \infty} \frac{R(N)}{\mathbf{E}(R(N))}=\lim _{N \rightarrow \infty} \frac{\gamma R(N) \log N}{2 \pi \sqrt{\gamma-1} N}=1 \quad \text { a.s. }
$$

As a special case from these results we recover the well-known Dvoretzky-Erdôs [13] results for the simple random walk on the plane (without the remainder term), as for the plane $L=1$ and $\gamma=2$. Thus we get

$$
\mathbf{E}(R(N)) \sim \frac{\pi N}{\log N}, \quad N \rightarrow \infty .
$$

and

$$
\lim _{N \rightarrow \infty} \frac{R(N)}{\mathbf{E}(R(N))}=\lim _{N \rightarrow \infty} \frac{R(N) \log N}{\pi N}=1 \quad \text { a.s. }
$$

Acknowledgement. The authors thank the referees for valuable comments and suggestions. Research supported by PSC CUNY Grant, No. 68080-0043 and by the Hungarian National Foundation for Scientific Research, No. K108615.

\section{References}

1. Bertacchi, D.: Asymptotic behaviour of the simple random walk on the 2-dimensional comb. Electron. J. Probab. 11, 1184-1203 (2006)

2. Bertacchi, D. and Zucca, F.: Uniform asymptotic estimates of transition probabilities on combs. J. Aust. Math. Soc. 75, 325-353 (2003)

3. Bertoin, J.: Iterated Brownian Motion and stable (1/4) subordinator. Statist. Probab. Letters 27, 111-114 (1996)

4. Chen, X.: How often does a Harris recurrent Markov chain recur? Ann. Probab. 27, 1324-1346 (1999)

5. Csáki, E., Csörgő, M., Földes, A. and Révész, P.: Strong limit theorems for a simple random walk on the 2-dimensional comb. Electron. J. Probab. 14, 2371-2390 (2009)

6. Csáki, E., Csörgő, M., Földes, A. and Révész, P.: On the supremum of iterated local time. Publ. Math. Debrecen 76, 255-270 (2010)

7. Csáki, E., Csörgő, M., Földes, A. and Révész, P.: On the local time of random walk on the 2-dimensional comb. Stochastic Process. Appl. 121, 1290-1314 (2011)

8. Csáki, E., Csörgő, M., Földes, A. and Révész, P.: Random walk on half-plane half-comb structure. Annales Mathematicae et Informaticae 39, 29-44 (2012)

9. Csáki, E., Csörgő, M., Földes, A. and Révész, P.: Strong limit theorems for anisotropic random walks on $\mathbb{Z}^{2}$. Periodica Math. Hungar. 67, 71-94 (2013)

10. Csáki, E., Földes, A. and Révész, P.: Strassen theorems for a class of iterated processes. Trans. Amer. Math. Soc. 349, 1153-1167 (1997)

11. Darling, D. A. and Kac, M.: On occupation times for Markoff processes. Trans. Amer. Math. Soc. 84, 444-458 (1957)

12. den Hollander, F.: On three conjectures by K. Shuler. J. Statist. Physics 75, 891-918 (1994)

13. Dvoretzky, A. and Erdős, P.: Some problems on random walk in space. Proc. Second Berkeley Symposium, pp. 353-367 (1951)

14. Heyde, C. C.: On the asymptotic behaviour of random walks on an anisotropic lattice. $J$. Statist. Physics 27, 721-730 (1982)

15. Heyde, C. C.: Asymptotics for two-dimensional anisotropic random walks. In: Stochastic Processes. Springer, New York, pp. 125-130 (1993) 
16. Heyde, C. C., Westcott, M. and Williams, E. R.: The asymptotic behavior of a random walk on a dual-medium lattice. J. Statist. Physics 28, 375-380 (1982)

17. Horváth, L.: Diffusion approximation for random walks on anisotropic lattices. J. Appl. Probab. 35, 206-212 (1998)

18. Lévy, P.: Processus Stochastiques et Mouvement Brownien. Gauthier Villars, Paris (1948)

19. Krishnapur, M. and Peres, Y.: Recurrent graphs where two independent random walks collide finitely often. Electron. Comm. Probab. 9, 72-81 (2004)

20. Nándori, P.: Number of distinct sites visited by a random walk with internal states. Probab. Th. Rel. Fields 150, 373-403 (2011)

21. Nash-Williams C. ST. J. A: Random walks and electric currents in networks. Proc. Cambr. Phil. Soc. 55, 181-194 (1959)

22. Petrov, V. V.: Limit Theorems of Probability Theory. Sequences of Independent Random Variables. Clarendon Press, Oxford (1995)

23. Révész, P.: Random Walk in Random and Non-Random Environments, 2nd ed. World Scientific, Singapore (2005)

24. Revuz, D.: Markov Chain. North-Holland, Amsterdam (1975)

25. Roerdink, J. and Shuler, K. E.: Asymptotic properties of multistate random walks. I. Theory. J. Statist. Physics 40, 205-240 (1985)

26. Seshadri, V., Lindenberg, K. and Shuler, K. E.: Random walks on periodic and random lattices. II. Random walk properties via generating function techniques. J. Statist. Physics 21, 517-548 (1979)

27. Shuler, K. E.: Random walks on sparsely periodic and random lattices I. Physica A 95, 12-34 (1979)

28. Silver, H., Shuler, K. E. and Lindenberg, K.: Two-dimensional anisotropic random walks. In: Statistical mechanics and statistical methods in theory and application (Proc. Sympos., Univ. Rochester, Rochester, N.Y., 1976), Plenum, New York, pp. 463-505 (1977)

29. Weiss, G. H. and Havlin, S.: Some properties of a random walk on a comb structure. Physica A 134, 474-482 (1986)

30. Westcott, M.: Random walks on a lattice. J. Statist. Physics 27, 75-82 (1982)

31. Woess, W.: Random Walks on Infinite Graphs and Groups, Cambridge Tracts in Mathematics, Vol. 138, Cambridge University Press, Cambridge (2000) 